\section{BMJ Paediatrics Open}

\title{
Task-specific gross motor skills training for ambulant school-aged children with cerebral palsy: a systematic review
}

Rachel Toovey, ${ }^{1,2}$ Charmaine Bernie, ${ }^{1,3}$ Adrienne R Harvey, ${ }^{1,3}$ Jennifer L McGinley, ${ }^{2}$ Alicia J Spittle 2,4

To cite: Toovey R, Bernie C, Harvey AR, et al. Task-specific gross motor skills training for ambulant school-aged children with cerebral palsy: a systematic review. BMJ Paediatrics Open 2017;1:e000078. doi:10.1136/ bmjpo-2017-000078

- Additional material is published online only. To view, please visit the journal online (http://dx.doi.org/10.1136/ bmjpo-2017-000078).

Received 11 May 2017 Revised 6 July 2017 Accepted 8 July 2017

\section{(a) CrossMark}

${ }^{1}$ Developmental Disability and Rehabilitation Research, Murdoch Children's Research Institute, Melbourne, Victoria, Australia

2Department of Physiotherapy, University of Melbourne, Melbourne, Victoria, Australia ${ }^{3}$ Department of Neurodevelopment and Disability, The Royal Children's Hospital, Melbourne, Victoria, Australia

${ }^{4}$ Victorian Infant Brain Studies, Murdoch Children's Research Institute, Melbourne, Victoria, Australia

Correspondence to Rachel Toovey; rachel.toovey@ mcri.edu.au

\section{ABSTRACT}

Objectives The primary objective is to systematically evaluate the evidence for the effectiveness of task-specific training (TST) of gross motor skills for improving activity and/or participation outcomes in ambulant school-aged children with cerebral palsy (CP). The secondary objective is to identify motor learning strategies reported within TST and assess relationship to outcome.

Design Systematic review.

Method Relevant databases were searched for studies including: children with $\mathrm{CP}$ (mean age $>4$ years and $>60 \%$ of the sample ambulant); TST targeting gross motor skills and activity (skill performance, gross motor function and functional skills) and/or participation-related outcomes. Quality of included studies was assessed using standardised tools for risk of bias, study design and quality of evidence across outcomes. Continuous data were summarised for each study using standardised mean difference (SMD) and 95\% Cls.

Results Thirteen studies met inclusion criteria: eight randomised controlled trials (RCTs), three comparative studies, one repeated-measures study and one singlesubject design study. Risk of bias was moderate across studies. Components of TST varied and were often poorly reported. Within-group effects of TST were positive across all outcomes of interest in 11 studies. In RCTs, betweengroup effects were conflicting for skill performance and functional skills, positive for participation-related outcomes (one study: Life-HABITS performance SMD $=1.19,95 \% \mathrm{Cl}$ 0.3 to 2.07, $p<0.001$; Life-HABITS satisfaction $S M D=1.29$, $95 \% \mathrm{Cl} 0.40$ to $2.18, p=0.001$ ), while no difference or negative effects were found for gross motor function. The quality of evidence was low-to-moderate overall. Variability and poor reporting of motor learning strategies limited assessment of relationship to outcome.

Conclusions Limited evidence for TST for gross motor skills in ambulant children with $\mathrm{CP}$ exists for improving activity and participation-related outcomes and recommendations for use over other interventions are limited by poor study methodology and heterogeneous interventions.

Registration PROSPERO ID42016036727

\section{INTRODUCTION}

Cerebral palsy (CP) is an umbrella term used to describe a group of disorders of movement which cause varying degrees of activity

\section{What is already known on this topic?}

Strong evidence for motor interventions involving task specificity for functional mobility in adults poststroke and for upper limb function in children with cerebral palsy exists.

- The effectiveness of task-specific gross motor skills training in ambulant school-aged children with cerebral palsy has not been systematically evaluated or synthesised.

\section{What this study hopes to add?}

A low-to-moderate overall quality of evidence was found for task-specific gross motor skills training for ambulant school-aged children with cerebral palsy.

- Limited evidence for task-specific training to improve specific skills performance, functional skills and participation-related outcomes exists.

- While clear recommendations for use of taskspecific training over other interventions are limited, ways to strengthen the evidence in future studies are identified.

limitations. ${ }^{1}$ The most widely used means for classifying gross motor function in children with CP is the Gross Motor Function Classification System (GMFCS) ${ }^{2}$ Children classified GMFCS I are able to walk and run but have limitations with speed, balance and coordination while children classified GMFCS V are transported in a wheelchair in all settings. Although the focus of the GMFCS is on functional mobility, the realm of gross motor activities, that is skills involving movement of the large muscles of the limbs or whole body, undertaken by children is much broader. ${ }^{3}$ Development of gross motor skills underpins functional, play and social activities across childhood and complex movement skills required for sports in older children. ${ }^{3}$ In children with CP, limitations in gross motor function increase as GMFCS level increases, however, children at all GMFCS levels (I-V) 
participate, on average, less in physical activities than their typically developing peers. ${ }^{2}$ This is an issue because of the known poor health outcomes in adulthood due to inactivity in childhood. ${ }^{4}$ Effective interventions tailored to GMFCS levels and developmental stages are required to improve these outcomes in this population.

The International Classification of Functioning, Disability and Health (ICF) has become the common language for clinicians, researchers and families for understanding the effect of $\mathrm{CP}$ on the individual and for targeting interventions. ${ }^{5} 6$ Where interventions previously focused on remediating limitations in body structures and functions, there has been a more recent acknowledgement of the importance of the effect of interventions within the activity and participation domains. ${ }^{78}$ Clinicians working with children with $\mathrm{CP}$ need guidance from evidence synthesis to implement effective means of improving physical skills and improve the uptake of these skills in the child's daily life. The historical bias towards impairment-focused motor interventions yielded few effective treatments, ${ }^{6910}$ thus more functional approaches have emerged.

Task-specific training (TST) involves practice of context-specific tasks where the intervention focuses on the skills needed for a task $(\mathrm{s})^{11}$ - there is similarity between the training task and the goal of the intervention. Although level I evidence exists for TST to improve gross motor activities in adults after stroke, ${ }^{12}$ the majority of high-level evidence for interventions involving task specificity in children with CP relates to training of upper limb or fine motor activities ${ }^{613}$ with limited evidence for gross motor skills training. TST inherently involves principles of motor learning with components including context, practice and dosage. ${ }^{11}$ Other motor learning strategies, such as feedback and task modification, have the potential to optimise TST, however, this has not been systematically studied. ${ }^{14}$ TST should involve varied components depending on the requirements of the skill, the environment and the function of the child. ${ }^{15}$ Moreover, training for a child of higher-level motor function (eg, GMFCS I-III) should be targeted towards different skills compared with training with a child of lower-level motor function (eg, GMFCS IV-V). Similarly, children of different ages and developmental stages have varying learning capabilities, and physical demands placed on them by their context. ${ }^{16}$

Previous systematic reviews of motor interventions in children with CP have been broad in terms of ages (including infants and children) and motor function (all GMFCS levels), and included interventions have been heterogeneous. ${ }^{1718}$ TST may be a promising approach for ambulant children who have specific gross motor skills goals, however, there has been no systematic review to examine the effectiveness of this approach in this population. The primary aim of this study is to evaluate and synthesise the evidence for the effectiveness of task-specific gross motor skills training in ambulant children aged 4-18 years with CP for activity and participation outcomes. The secondary aim of this study is to identify motor learning strategies reported within TST and assess relationship to outcome.

\section{METHOD}

\section{Eligibility criteria}

Published studies were included if they met all of the following criteria:

1. Level of evidence: all group design studies categorised as level II-IV using the American Academy of Cerebral Palsy and Developmental Medicine (AACPDM) levels of evidence ${ }^{19}$ and studies classified as level I single-subject designs involving over 10 participants.

2. Population: the majority (ie, $>60 \%$ ) of the participants were ambulant children with CP (GMFCS IIII), and the mean sample age was 4-18 years.

3. Interventions: TST of gross motor skills where there was similarity between the training task and the goal of the intervention, including those interventions described as involving motor learning strategies/coaching, goal-directed training, activity focused training and/or functional skills training. Any duration or intensity of TST.

4. Comparison: studies comparing TST with another intervention, another type of TST or no intervention.

5. Outcomes: activity outcomes including gross motor skill performance (specific to the task being trained or other gross motor task to assess for transferability), gross motor function and functional skills; and participation-related outcomes. Only studies reporting outcomes separately for children with CP.

Exclusion criteria: TST was applied within a combined intervention approach and the influence of TST could not be isolated (eg, botulinum toxin-A, virtual reality, treadmill training, orthoses or robotics), $>10 \%$ of the intervention was passive or the article was not in English.

\section{Search strategy}

Relevant articles were identified from Medline, EMBASE, CINAHL, PsycINFO, SPORTDiscus and PubMed with all searches limited to articles published in peer-reviewed journals in English. A search was conducted in June 2016 and search terms were tailored for each database. Reference lists of included studies and related reviews were also searched. See online supplementary appendix 1 for full search strategy for Ovid databases (Medline, EMBASE and PubMed).

\section{Study selection, data extraction and quality assessment}

Study selection, data extraction and quality assessment was completed by two authors (RT and $\mathrm{CB}$ ) independently, with a third author (AH, JM or AS) to resolve any disagreements. If inclusion was uncertain from abstract, the full-text was retrieved.

For included studies, data were extracted using a customised form based on the Cochrane recommendations. ${ }^{20}$ Data extracted included: study details (author, 
year, country, funding), study design, AACPDM level of evidence, ${ }^{19}$ study sample characteristics, detailed TST characteristics (including motor learning strategies), comparison intervention characteristics, outcomes measured and associated ICF domain, effects of the intervention and conclusions. Reported motor learning strategies in all TST, including when studies involved a comparison of two or more TST approaches, were identified using predefined motor learning strategy codes based on current literature. ${ }^{21} 22$ The authors were contacted for complete data extraction when needed.

Risk of bias for individual studies was assessed using the Cochrane Risk of Bias Tool. ${ }^{20}$ Overall risk of bias was determined by predominant level of bias (at least four of the seven domains) across the domains of the Risk of Bias Tool. Intervention replicability was assessed using relevant components of the Template for Intervention Descriptions and Replication (TIDieR) Checklist. ${ }^{23}$ The Grading of Recommendations Assessment Development and Evaluation (GRADE) system was used to assess the quality of the evidence across outcomes. ${ }^{24}$

\section{Narrative synthesis and data analysis}

Study design and AACPDM level of evidence were used for narrative synthesis. Higher-level group designs (AACPDM levels II-III) were reported together, while lower-level group designs (IV) and single-subject design studies were reported separately. Within the level IIIII group designs, studies were considered as either 'TST versus a comparison' or 'TST versus TST'. Finally, within each grouping, activity outcomes were organised into three constructs: gross motor skill performance, functional skills and gross motor function while participation-related outcomes were grouped together. Gross motor skill performance outcomes included those measuring a specific gross motor skill (eg, walking or throwing), functional skills outcomes included those measuring a broader range of skills in domains related to function (eg, self-care or social skills), while gross motor function outcomes included those measuring a range of gross motor-related skills.

Analyses were conducted using Cochrane Review Manager Software RevMan V.5.3. Only data from level II studies comparing TST with comparison interventions were included in quantitative analysis to ensure comparison was between studies of similar design. Continuous data were summarised for each study within outcomes of interest using standardised mean difference (SMD) and 95\% CIs. An SMD of 0.2 was considered small, 0.4-0.6 moderate and 0.8 a large effect size. ${ }^{20}$ Given the heterogeneity between studies in tasks and characteristics of the TST and comparison interventions, meta-analysis was not undertaken.

\section{RESULTS}

Following removal of duplicates, the abstracts of 1247 studies were screened and 145 full-text articles were retrieved for full appraisal (figure 1). Thirteen studies involving 405 participants met inclusion criteria and underwent narrative synthesis with six of these studies involving 237 individuals included in data analysis. Characteristics of included studies are summarised in table 1 .

The 13 studies included 12 group designs and one single-subject design (level I). The group designs involved eight randomised controlled trials (RCTs) (level II), three comparative studies with concurrent controls (level III) and one repeated measures study (level IV). Eleven studies did not report adverse events as an outcome and two studies reported no adverse events. ${ }^{25} 26$

\section{Risk of bias assessment}

Risk of bias was low in four studies, ${ }^{1427-30}$ unclear in seven studies $^{1425} 26$ 31-34 and high in two studies ${ }^{34-36}$ leading to an overall moderate risk of bias across the studies (table 2). Major sources of bias included: studies without

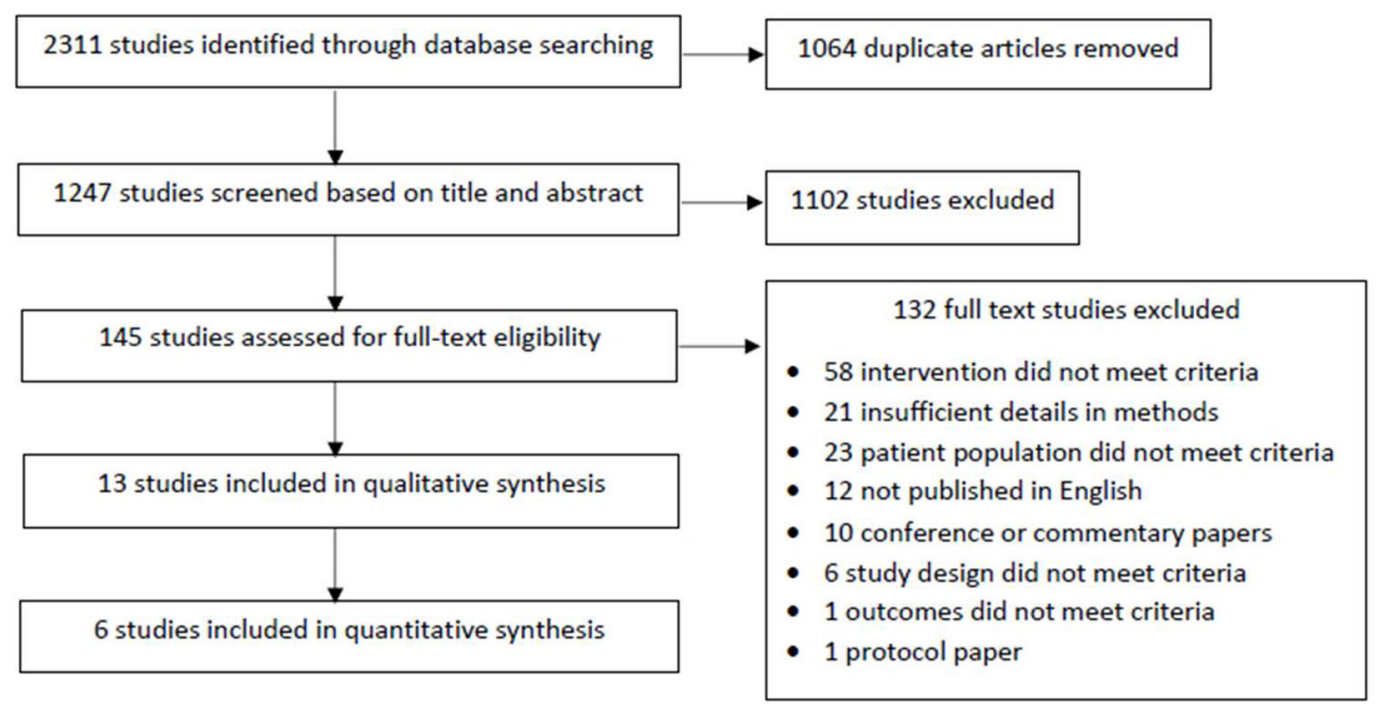

Figure 1 Preferred Reporting Items for Systematic Reviews and Meta-Analyses (PRISMA) flow chart of included and excluded studies. 


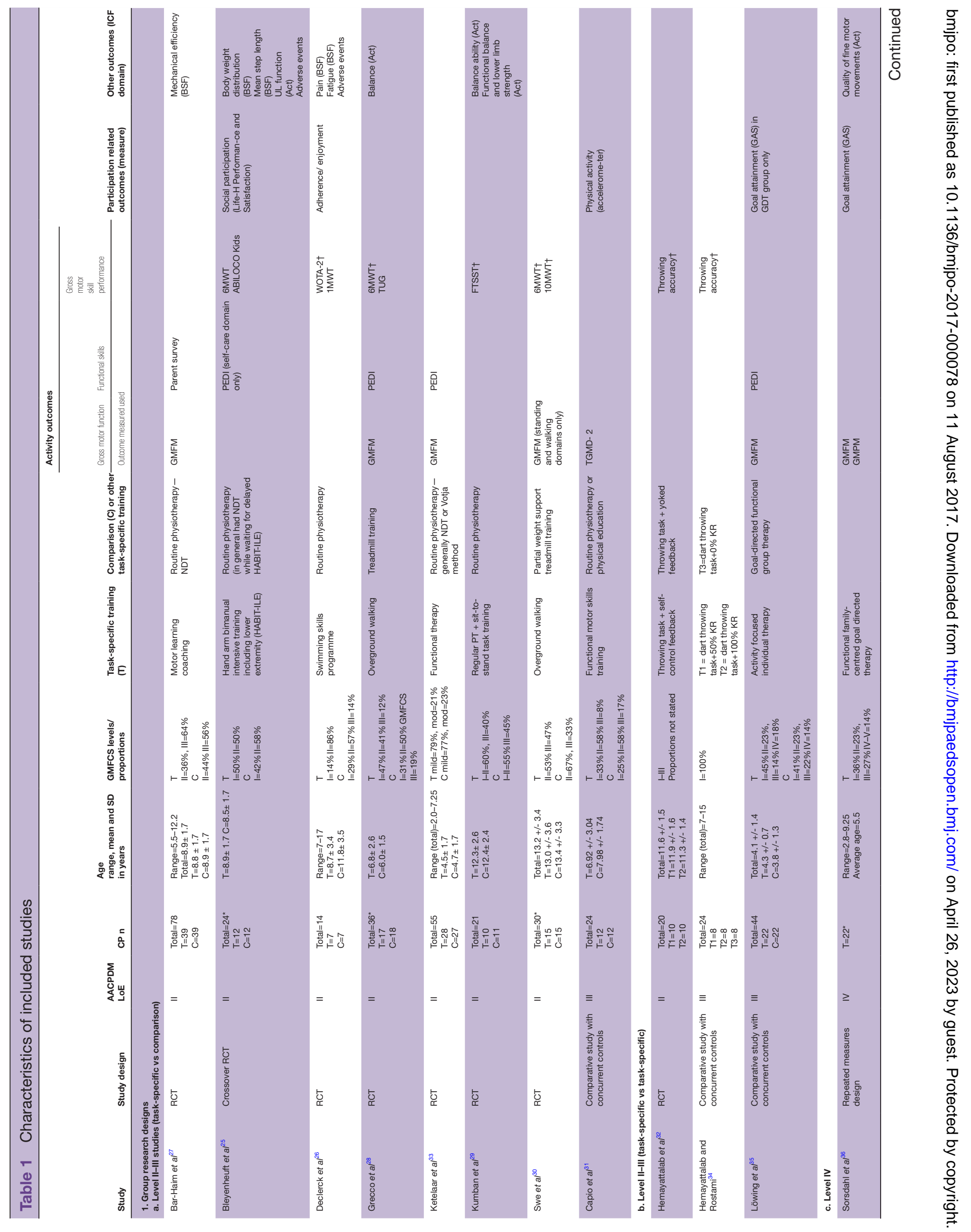




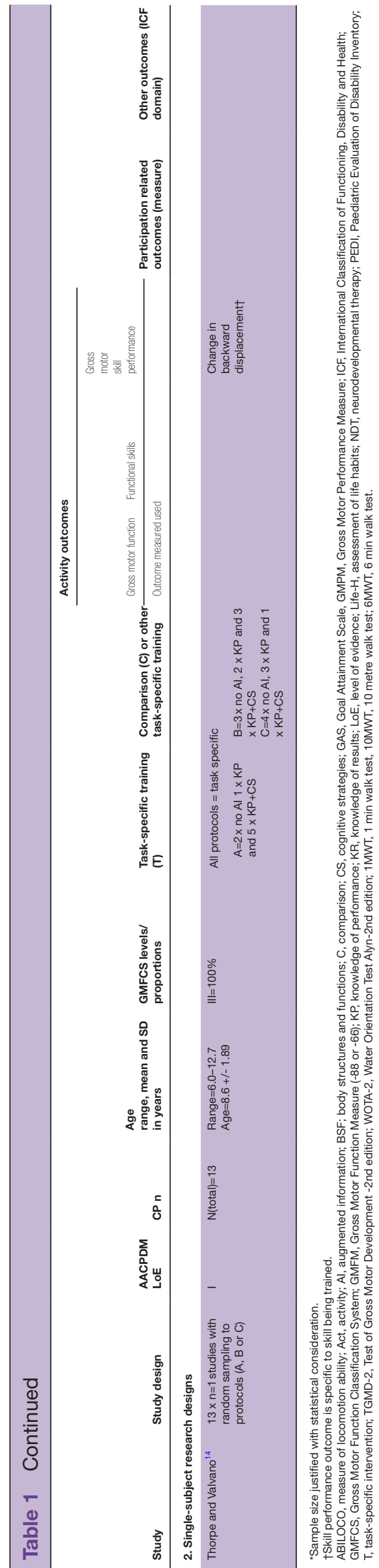

randomisation or allocation concealment, a lack of participant/personnel blinding and incomplete outcome data. No study reported the use of intention-to-treat analysis and reporting of outcome assessor blinding was inconsistent. Poor reporting of study conduct limited assessment of risk of bias in the seven studies with unclear risk of bias.

\section{Level II-III group designs}

Task-specific training versus comparison Interventions

Seven RCTs and one comparative study ${ }^{31}$ (table 1) compared TST with another intervention. The target of TST varied; four studies involved training a variety of tasks or movement skills, ${ }^{21} 253133$ one focused on swimming ${ }^{26}$ and another trained sit to stand. ${ }^{29}$ Six studies compared TST with 'routine physiotherapy'. ${ }^{25-27} 293133$ This was generally poorly defined; described as neurodevelopmental therapy (NDT) ${ }^{252733}$ in three studies, physical education in one study ${ }^{31}$ or not reported..$^{26} 29$ The remaining two studies involved overground walking compared with treadmill training; with overground walking deemed as the TST, as treadmill training was considered not specific to the gross motor aim of improving overground walking.

Two of the eight studies were goal-directed while four studies reported the TST was driven by motor learning principles $^{25} 273133$ (table 3). Repetitive practice was the most commonly reported motor learning strategy with feedback and task modification in four ${ }^{25} 272833$ and five 2527293133 studies, respectively. Physiotherapists generally conducted interventions but characteristics varied widely. Overall intervention time ranged from $3^{31}$ to 90 hours $^{25}$ over periods from 10 days $^{25}$ to 6 months. ${ }^{33}$ Reported settings were largely ecological while format was group-based in two studies, ${ }^{25} 26$ otherwise was not stated.

\section{Outcomes and effects}

All eight studies reported outcomes at the activity level, three reported participation-related outcomes and three involved body structure and function outcomes (table 1). Outcomes were measured at one to three time points. In general, T1 was immediately following the intervention, while T2 (4 weeks-6 months) $)^{25-30} 33$ and T3 (2-12 months ${ }^{2633}$ were varied.

\section{Activity outcomes}

Gross motor skill performance was measured in five of the eight studies ${ }^{252628-30}$ with four measuring skill performance specific to the training tasks ${ }^{26}{ }^{28-30}$ (table 1). Overall, skills and measures were varied. Four studies measured walking performance ${ }^{25262830}$ : using the $6 \mathrm{~min}$ walk test $(6 \mathrm{MWT})^{2528}{ }^{30}$ or the timed up and go, $1 \mathrm{~min}$ walk test, 10 metre walk test and the ABILOCO Kids questionnaire..$^{252628} 30$ Between-group effects of TST on walking performance were mixed (figure 2A-B). Large effects favouring TST on walking performance at T1 were 


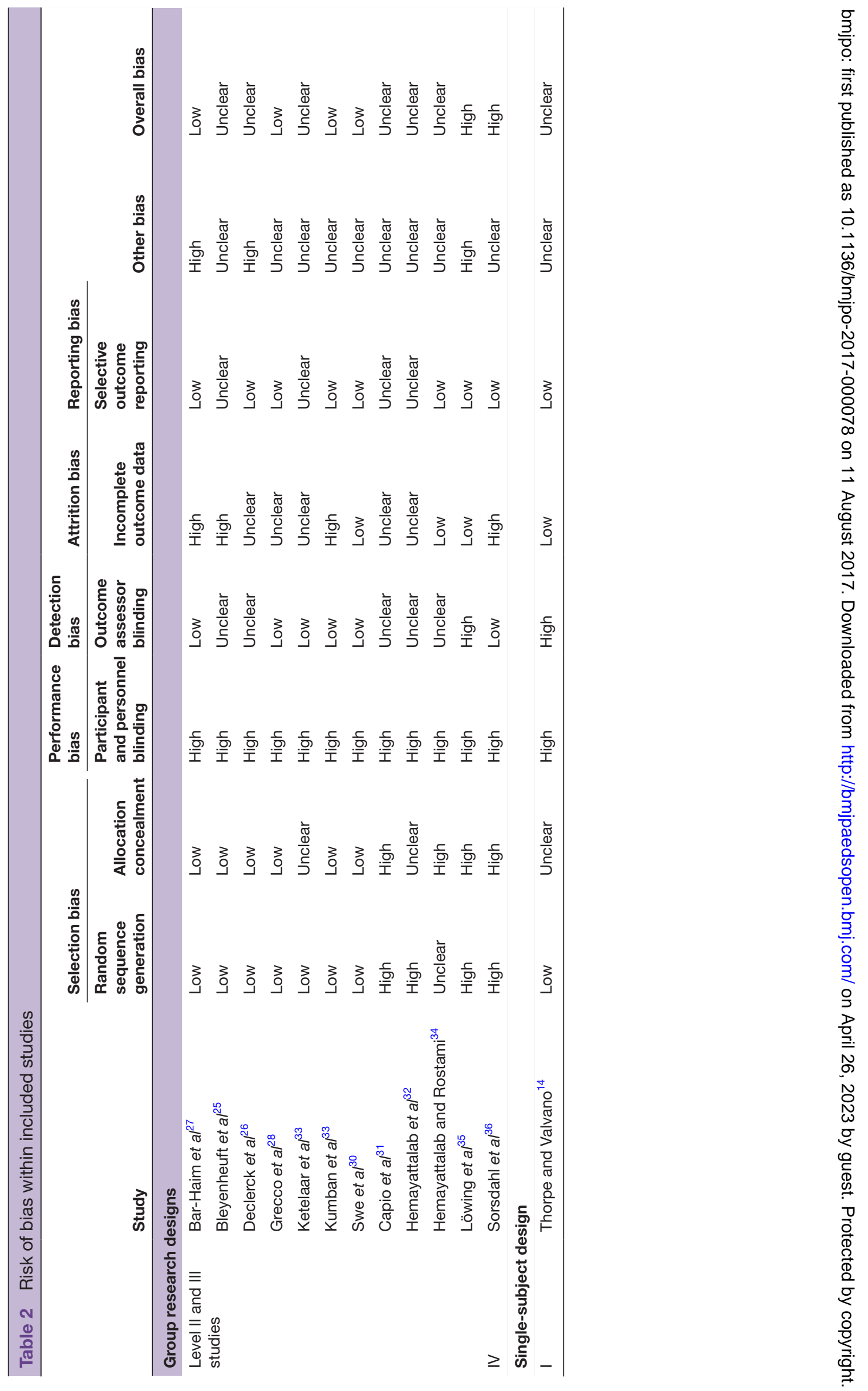




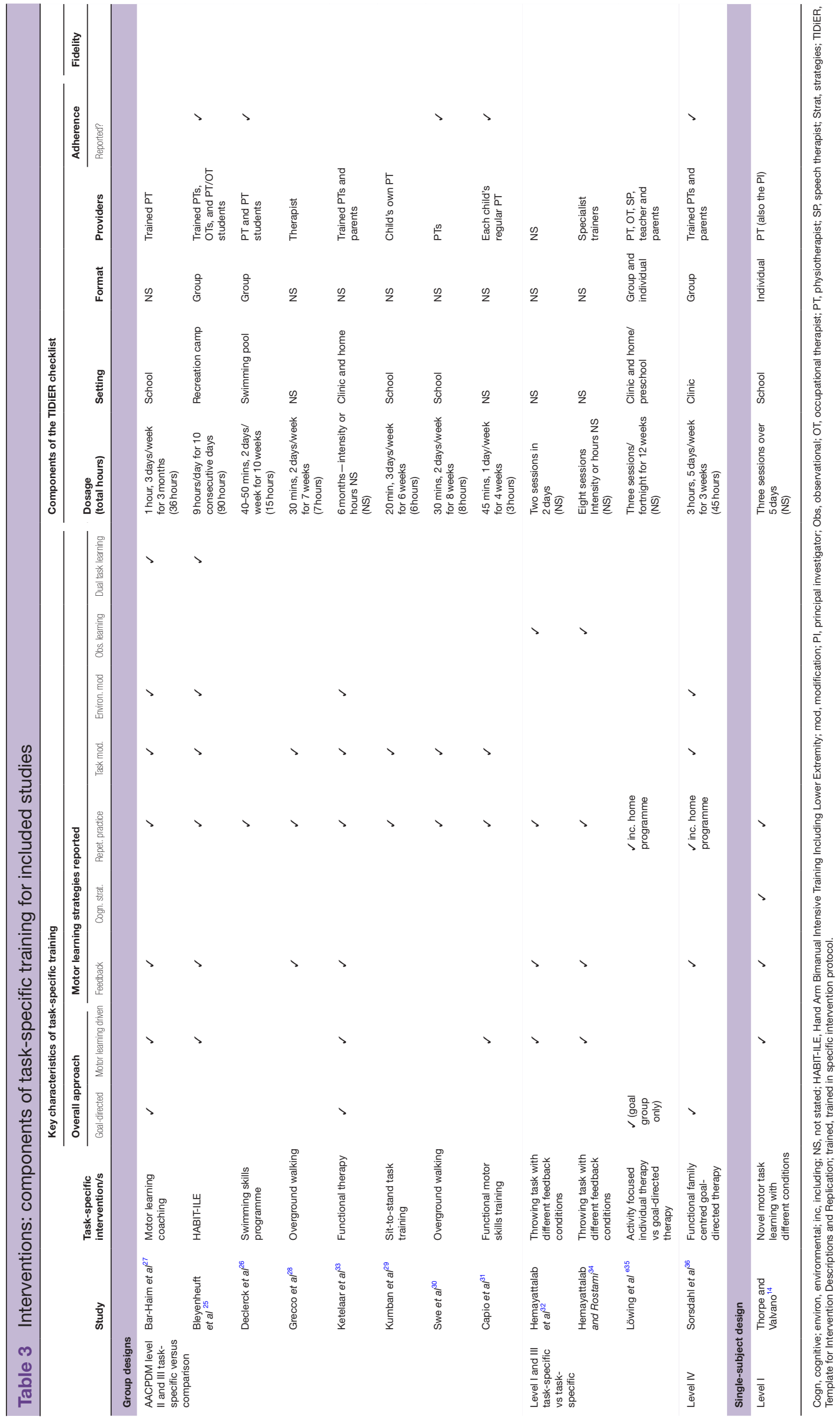




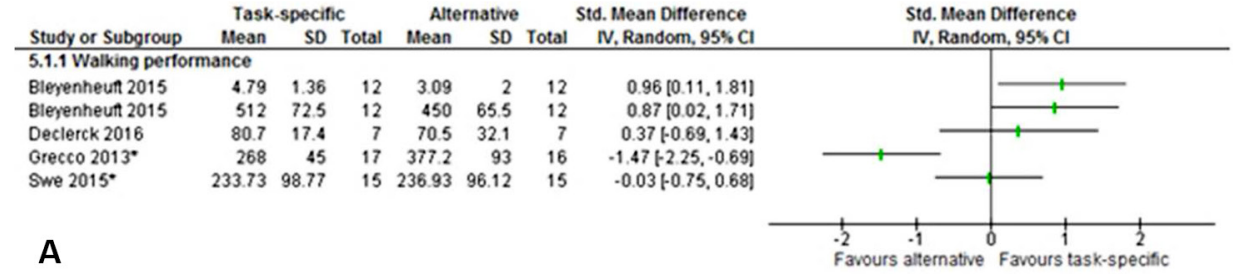

found (ABILOCO Kids SMD $=0.96,95 \% \mathrm{CI}=0.11$ to 1.81 ; 6MWT SMD $=0.87,95 \%$ CI 0.02 to 1.71$)$ by Bleyenheuft et al (Hand Arm Bimanual Intensive Training Including Lower Extremity vs routine physiotherapy). ${ }^{25}$ However, large negative effects at both T1 (6MWT SMD $=-1.47$, $95 \% \mathrm{CI}-2.25$ to -0.69$)$ and at 4 weeks follow-up (6MWT $\mathrm{SMD}=-1.46,95 \% \mathrm{CI}-2.24$ to -0.68$)$ were found by Grecco et al (overground walking vs treadmill training). ${ }^{28}$ Non-significant mixed effects were found at both time points in the remaining two studies. ${ }^{2630}$ Swimming performance was measured using the Water Orientation Test Alyn-2nd edition in one study ${ }^{26}$ and sit-to-stand performance was measured using the five-times sit-to-stand test in another. ${ }^{29}$ There was no between-group effect found for TST on sit-to-stand ${ }^{29}$ or swimming performance at $\mathrm{T} 1{ }^{26}$ Within-group effects of TST were positive for all skill performance outcomes in each study, except for the GMFCS III subgroup in one study. ${ }^{29}$

Overall gross motor function was measured in five of the eight studies 2728303133 (table 1). Four studies ${ }^{27283033}$ used the Gross Motor Function Measure (GMFM) ${ }^{37}$ Three studies measured all domains of the $\mathrm{GMFM}^{27} 2833$ (A: lying and rolling, B: sitting, C: crawling and kneeling, D: standing, $\mathrm{E}$ walking running and jumping), while one study measured domains D and E only. ${ }^{30}$ Nil or negative effect of TST was found at T1 or at T2 (figure 3a-d) 27283033 on domains $\mathrm{D}$ and $\mathrm{E}$. Three studies demonstrated no effect of TST at T1 or T2 in either domain, ${ }^{27} 3033$ while the fourth study (Grecco et al) showed a large negative effect for both domains at T1 (GMFM-D SMD $=-1.39$, 95\% CI -2.16 to -0.62 ; GMFM-E SMD $=-1.97,95 \%$ CI -2.82 to -1.12 ) and T2 (GMFM-D SMD $=-1.32,95 \% \mathrm{CI}$ -2.09 to -0.56 ; GMFM-E SMD $=-2.08,95 \%$ CI -2.95 to -1.22).$^{28}$ No significant effects of TST were found on total GMFM score (figure 3E-F) ${ }^{27} 33$. Again, a large effect favouring the comparison was found for total score at both time points (T1 SMD $=-1.83,95 \%$ CI -2.63 to -1.03 ; T2 $\mathrm{SMD}=-1.67,95 \% \mathrm{CI}-2.48$ to -0.87 ) by Grecco $e t$ al. $^{28}$ The remaining level III design study reported a significant positive effect of TST on gross motor function as measured by the Test of Gross Motor Development-2nd edition. ${ }^{31}$ Within-group effects of TST on gross motor function were positive in all five studies.

Functional skill outcomes were measured in four of the eight studies ${ }^{25} 272833$ (table 1). Functional skills included self-care, assessed by the self-care domain of the Paediatric Evaluation of Disability Inventory (PEDI), in three studies $^{25} 28{ }^{33}$; mobility, measured by the PEDI mobility domain in two studies ${ }^{28} 33$ and a parent questionnaire ${ }^{27}$; and social function assessed by the PEDI social function domain in one study. ${ }^{28}$ A large effect (SMD $=1.07,95 \%$ CI 0.21 to 1.94) favouring TST was found for self-care skills at $\mathrm{T} 1$ in the study by Bleyenheuft et al. ${ }^{25}$ No significant effects were found for the other two studies measuring self-care at T1 and T228 ${ }^{28} 33$ (figure 4A-B). No effect of TST on mobility skills (PEDI mobility domain) was found at T1 (figure 4C), but a moderate effect was found at 6 months (T2 SMD $=0.58,95 \%$ CI 0.04 to 1.12 ) and a moderate-tolarge effect found at 12-month follow-up (T3 SMD=0.76, $95 \%$ CI 0.21 to 1.31 ) by Ketelaar et al (functional therapy vs routine physiotherapy). ${ }^{33}$ In contrast, a large negative effect (SMD $=-1.32,95 \%$ CI -2.09 to -0.56 ) was found for mobility skills at $\mathrm{T} 1$ by Grecco $e t a l^{28}$ (figure 4C). No effect on mobility on the parent questionnaire was found at T1 or 6 months post ${ }^{27}$ or on social function $\mathrm{T} 1$ or at 4 weeks follow-up. ${ }^{28}$ Within-group effects of TST on all functional skills outcomes were positive in all four studies.

\section{Participation-related outcomes}

Participation-related outcomes were measured in only three of the eight studies ${ }^{25} 2631$ (table 1). Measures included social participation using the Life-HABITS performance and satisfaction questionnaire,${ }^{25}$ adherence and enjoyment ${ }^{26}$ and participation in physical activity using an accelerometer. ${ }^{31}$ Large effects favouring TST were found for both social participation performance (SMD $=1.19,95 \%$ CI 0.31 to 2.07 ) and satisfaction $(\mathrm{SMD}=1.29,95 \%$ CI 0.40 to 2.18$)$ by Bleyenheuft et al. ${ }^{25}$ Positive effects of TST on weekend physical activity were reported at $\mathrm{T} 1 .^{31}$ High adherence rates and enjoyment 


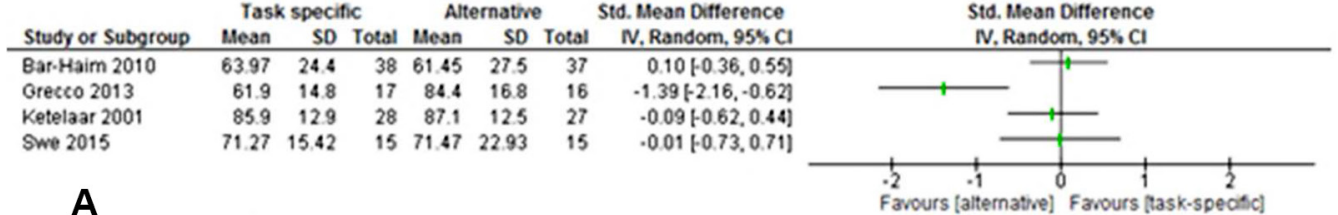

A

Favours [alternative] Favours [task-specific)

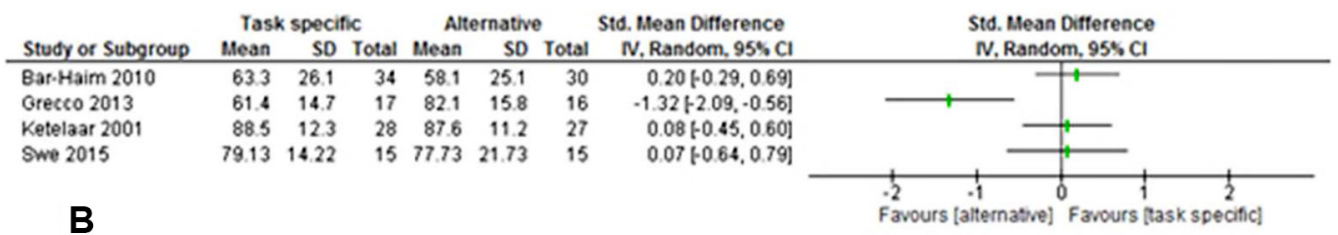

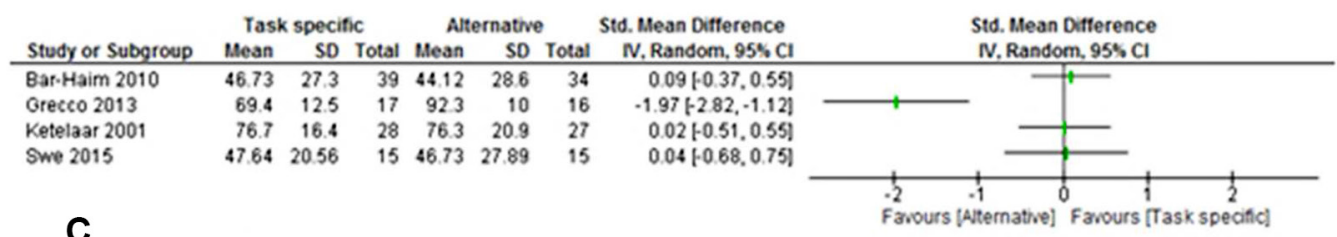

C

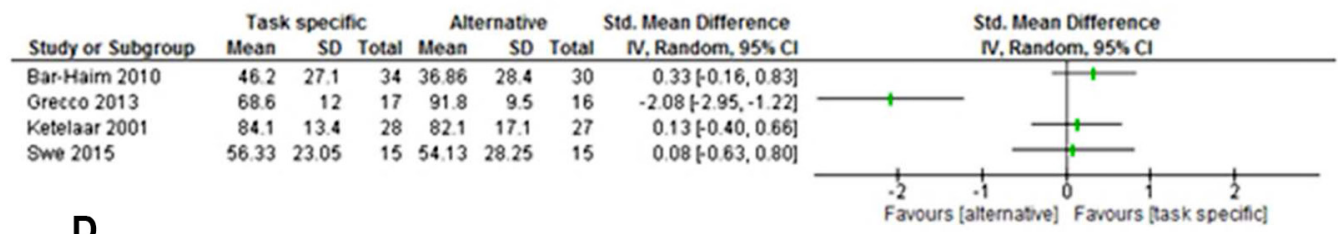

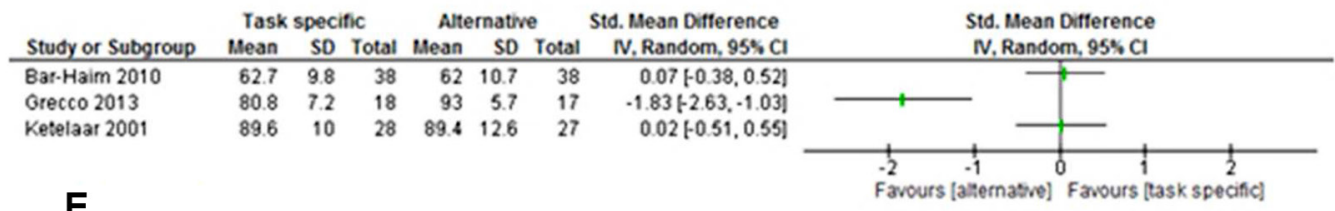

$\mathbf{E}$

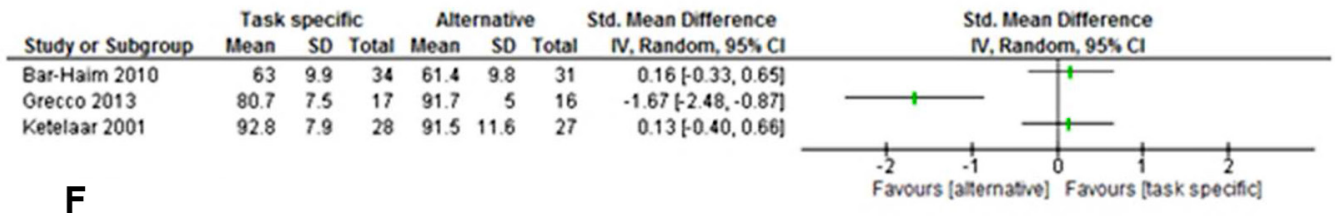

Figure 3 (A) Gross motor function - task specific vs alternative: standing ability (Gross Motor Function Measure (GMFM) domain D) at T1. (B) Gross motor function - task specific vs alternative: standing ability (GMFM domain D) at T2. (C) Gross motor function-task specific vs alternative: walking, running and jumping ability (GMFM domain E) at T1. (D) Gross motor function-task specific vs alternative: walking, running and jumping ability (GMFM domain E) at T2. (E) Gross motor functiontask specific vs alternative: overall GMFM score at T1. (F) Gross motor function-task specific vs alternative: overall GMFM score at $\mathrm{T} 2$.

levels for TST were also reported but without comparison group data. ${ }^{26}$

\section{Task-specific versus task-specific \\ Interventions}

Three studies compared two or more task-specific interventions including one RCT (level II) ${ }^{32}$ and two comparative studies (level III). ${ }^{34} 35$ Two studies ${ }^{32} 34$ compared the effect of TST programmes with different feedback conditions on a throwing task (table 1). The throwing task training was informed by motor learning principles but was not goal-directed (table 3). The intensity of TST in both studies was approximately 2 hours over 2 days. ${ }^{32}{ }^{34}$ The other study examined the effects of goal-directed TST compared with activity-based TST, ${ }^{35}$ with 18 sessions over 12 weeks with parent involvement across various settings. ${ }^{35}$ The goal-directed programme involved group and individual sessions while the activity programme was individual only.

\section{Outcomes}

Gross motor skill performance (specific to the trained task), assessed by throwing accuracy, was the sole outcome in both throwing studies. ${ }^{32} 34$ This was assessed immediately postintervention (T1-acquisition) in both studies, 24 hours later in one ${ }^{32}$ (T2-retention) and 3 days later 

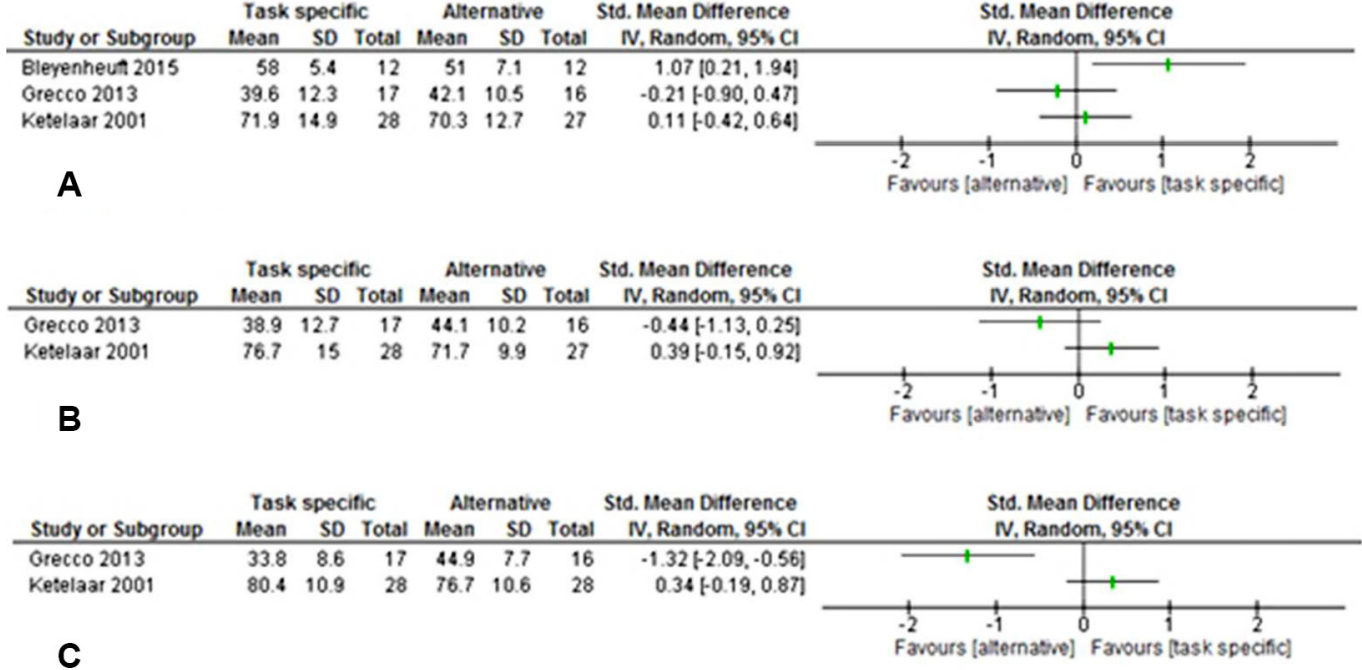

Figure 4 (A) Functional skills - task specific vs alternative: self-care at T1. (B) Functional skills - task specific vs alternative: self-care at T2. (C) Functional skills-task specific vs alternative: mobility skills at T1.

(T2) in the other. ${ }^{34}$ Outcomes were assessed at T1 only in the remaining study: including gross motor function (total GMFM score) and functional skills (all domains of the PEDI). The participation-related outcome, goal attainment, was assessed in the goal-directed group only by the Goal Attainment Scale (GAS). ${ }^{35}$

Knowledge of results provided every trial was found to improve throwing accuracy significantly more than 50\% or no feedback at T1. However, at T2, those receiving knowledge of results $50 \%$ of the time performed significantly better than those who received feedback every trial or no feedback at all. ${ }^{34}$ Self-controlled feedback was found to improve throwing accuracy significantly more than yoked feedback at T2 but not at T1 ${ }^{32}$ Goal-directed training improved overall gross motor function and the functional skills of mobility and self-care significantly more than activity-based training. ${ }^{35}$ There was no difference in social function between the groups. Eighty-five per cent of goals were attained with goal-directed TST. ${ }^{35}$

\section{Level IV group design}

One repeated measures study involving goal-directed, group-based, intensive TST was included ${ }^{36}$ (table 1 , table 3). Gross motor function (overall GMFM score), functional skills (mobility, self-care and social function using the PEDI) and goal attainment (GAS) were measured at three baseline time points, immediately (T1) and 3 weeks (T2) after the intervention. Positive effects were reported for overall gross motor function at both $\mathrm{T} 1$ and $\mathrm{T} 2$, and for self-care skills at $\mathrm{T} 2$, while $66 \%$ of goals were attained at $\mathrm{T} 2$.

\section{Single-subject design}

One level I single-subject design study involving randomisation to one of three feedback protocols for learning to move an exercise vehicle backward was included ${ }^{14}$ (table 1). Gross motor skill performance (specific to the trained task) was measured using backward displacement of the vehicle 2 days following the training (T1). Eight of the 13 participants demonstrated significant improvement at T1. No specific feedback protocol was clearly superior.

\section{Intervention replicability}

Reporting of intervention characteristics varied widely (table 3). Four studies reported intervention providers were trained in the intervention protocol, ${ }^{25} 273336$ five studies reported the format of the intervention ${ }^{1425} 263536$ while participant adherence was described in five studies. ${ }^{25} 26303136$ No study reported provider fidelity while comparison interventions were generally ambiguously reported.

\section{Quality of the evidence by outcome}

The overall quality of evidence was moderate for gross motor skill performance and functional skills and low for gross motor function and participation-related outcomes.

\section{DISCUSSION}

To our knowledge, this is the first systematic review to evaluate and synthesise the evidence for task-specific gross motor skills training in ambulant school-aged children with CP. Given the focus of TST is on practice of tasks rather than remediating impairments, and the increasing recognition of importance of child and family centred effects of interventions, this review explicitly focused on activity and participation outcomes. In general, positive within-group effects of TST were reported across outcomes of interest. However, in RCTs where TST was compared with comparison interventions, between-group effects were largely non-significant with the exception of two studies ${ }^{25} 28$ reporting large but conflicting effects. Overall, there was moderate quality evidence for conflicting effects of TST to improve specific 
skills performance and functional skills, but low quality evidence showing no difference or negative effects on gross motor function. For participation-related outcomes, low quality evidence for positive effects of TST was found. Positive effects across all outcomes were generally found immediately following TST, with evidence of long-term retention lacking.

The secondary aim of this study was to identify motor learning strategies reported in TST and assess any relationship to outcome. The overall poor reporting of motor learning strategies and heterogeneity in interventions and outcomes limited definitive conclusions. However, this review provides some insight into potentially important characteristics of interventions, in particular practice dosage, feedback and goals. Unsurprisingly, given its intrinsic relationship to TST, the most consistently reported motor learning strategy was repetitive practice. While reporting of dosage was variable, the largest positive effects of TST were found where dosage was highest. ${ }^{25}$ Although no specific feedback condition emerged as clearly superior in the three studies comparing these, ${ }^{1432} 34$ results suggest different feedback conditions may influence the phases of training differently. Replication of these studies for varying tasks is required to provide further clarity into the role of feedback. Although causal inferences are limited due to lack of randomisation, better activity outcomes were found when the TST was goal-directed..$^{35}$ These results are consistent with the growing evidence base for interventions targeted towards the goals of children with CP and their families. ${ }^{6} 16$

Our review found some conflicting results between studies, with large positive and negative effects found in the studies by Bleyenheuft $e t a l^{25}$ and Grecco $e t a l^{28}$ respectively. The TST in the study by Bleyenheuft $e t a l^{25}$ was high in dose, explicitly driven by motor learning principles and involved a wide range of gross motor tasks including ball skills, cycling and walking. By contrast, in the study by Grecco et $a l,^{28}$ dosage of TST (overground walking) was low in comparison and limited motor learning strategies were reported. The comparison treatment in this study was treadmill training without body weight support - training that may be regarded as task-related but was not deemed TST given the primary goal of the study was to improve overground walking. While practice dose was equal between the groups in the study by Grecco $e t a l,{ }^{28}$ task progression was potentially greater with the use of treadmill functions. These results suggest task-related adjuncts, such as treadmill training, may be more beneficial than pure TST for developing (rather than acquiring) gross motor skills, such as walking in this already-ambulant population.

\section{Limitations across the included studies}

Low-to-moderate quality of evidence was found across the outcomes due to significant methodological limitations in the studies, in addition to risk of bias. First, sample size calculation was not reported in most studies (table 1). Wide CIs found for outcome data from level II to III studies (figures 2-4) suggest inadequately powered samples may have reduced precision and thus limit the generalisability of the findings. Second, the limited detail in reporting potentially reduces the robustness of research findings if the study cannot be replicated, and limits implementation. Third, limitations in outcome measurement selection may have influenced the generalisability and transferability of some findings. The GMFM and the PEDI are a well-established tools for evaluating change in children with CP, ${ }^{38}$ and were used in six $x^{272830333536}$ and four studies ${ }^{252833} 35$, respectively. However, concerns have been raised about the responsiveness of these measures in higher functioning children, ${ }^{38}$ the target population of this review. While common use makes them appealing, their broad focus means skills targeted by the TST may not have been adequately captured. Finally, far fewer participation-related outcome measures were used compared with activity outcomes thus any evidence that improved activity through TST leads to improved participation is weak at best.

\section{Limitations of this review}

This review chose to narrow the inclusion criteria to ambulant school-aged children with CP, interventions of gross motor skill TST and activity and participation domains outcomes, in order to draw specific conclusions. This was not possible for all questions posed by this review due to study design issues and heterogeneity, which also precluded meta-analysis. Furthermore, the methodology of the review itself has some limitations. The impact of publication bias was not evaluated. Inclusion was limited to published articles in English meaning some studies, including grey literature, may have been missed. Furthermore, non-randomised studies were retained in this review. Although the Cochrane Risk of Bias tool and the GRADE system are the most widely used systems for assessing risk of bias and the quality of the evidence across outcomes, respectively, ${ }^{20}{ }^{24}$ these tools do emphasise randomised studies.

\section{Conclusions and recommendations}

Despite the largely positive within-group effects of TST over time across each outcome of interest, between-group effects were conflicting for skill performance and functional skills, positive for participation-related outcomes while no difference or negative effects were found for gross motor function. Given the low-to-moderate quality of this evidence, there is currently limited evidence to support task-specific gross motor skills training for improving these activity and participation-related outcomes in children with CP. Clear recommendations around whether TST is superior to other interventions cannot be made. Before conclusions can be made about any relationship of motor learning strategies to outcome, more consistent reporting and studies designed to test this are required. 
The importance of tailoring motor interventions to individual goals and lifelong physical activity is increasingly being recognised. ${ }^{49}$ Thus, to enable specific recommendations, strengthening the evidence base is imperative. Adequately powered samples, rigorous study design and consistent reporting with attention to reporting interventions to allow for reproducibility and appropriate evidence synthesis is required. Future challenges also include considering issues with outcome measure responsiveness and intervention heterogeneity, and optimising TST through the use of motor learning strategies.

\section{Twitter @RachelToovey, @AliciaSpittle}

Contributors All named authors contributed to the design of this review. RT collected data, carried out data analyses and led interpretation and reporting. CB collected data and contributed to interpretation and reporting. ARH, JLM and AJS contributed to data analysis, interpretation and reporting. All named authors approved the final manuscript as submitted.

Funding The research was funded by an Australian Government Research Training Program Scholarship (RT), National Health Medical Research Council (NHMRC) Centre of Research Excellence in Cerebral Palsy ID 1057997 (RT and AH), Centre of Research Excellence in Newborn Medicine ID 1060733 (AJS), NHMRC Career Development Fellowship ID 1053767 (AJS), Melbourne Children's Campus Clinician Scientist Fellowship (AH), Murdoch Children's Research Institute (RT, CB and AH) and the Victorian Government's Operational Infrastructure Support Program.

Competing interests None declared.

Provenance and peer review Not commissioned; externally peer reviewed.

Data sharing statement Data relating to the quantitative synthesis are available on request.

Open Access This is an Open Access article distributed in accordance with the Creative Commons Attribution Non Commercial (CC BY-NC 4.0) license, which permits others to distribute, remix, adapt, build upon this work non-commercially, and license their derivative works on different terms, provided the original work is properly cited and the use is non-commercial. See: http://creativecommons.org/ licenses/by-nc/4.0/

C) Article author(s) (or their employer(s) unless otherwise stated in the text of the article) 2017. All rights reserved. No commercial use is permitted unless otherwise expressly granted.

\section{REFERENCES}

1. Bax M, Goldstein M, Rosenbaum P, et al. Proposed definition and classification of cerebral palsy, April 2005. Dev Med Child Neurol 2005;47:571-6.

2. Palisano R, Rosenbaum P, Walter S, et al. Development and reliability of a system to classify gross motor function in children with cerebral palsy. Dev Med Child Neurol 1997;39:214-23.

3. Jones RA, Riethmuller A, Hesketh K, et al. Promoting fundamental movement skill development and physical activity in early childhood settings: a cluster randomized controlled trial. Pediatr Exerc Sci 2011;23:600-15

4. Fernandes RA, Zanesco A, Sansecso A. Early physical activity promotes lower prevalence of chronic diseases in adulthood. Hypertens Res 2010;33:926-31.

5. World Health Organisation. ICF: international classification of functioning, disability and health. Geneva: World Health Organisation, 2001.

6. Novak I, Mclntyre S, Morgan C, et al. A systematic review of interventions for children with cerebral palsy: state of the evidence. Dev Med Child Neurol 2013;55:885-910.

7. Imms C, Granlund M, Wilson $\mathrm{PH}$, et al. Participation, both a means and an end: a conceptual analysis of processes and outcomes in childhood disability. Dev Med Child Neurol 2017;59.

8. Rosenbaum P. Cerebral palsy: what parents and doctors want to know. BMJ 2003;326:970-4.

9. Valvano J. Activity-focused motor interventions for children with neurological conditions. Phys Occup Ther Pediatr 2004;24:79-107.
10. Gordon J. Assumptions Underlying Physical Therapy Intervention: Theoretical and Historical Perspectives. In: Carr J, Shepherd RB, eds. Movement Science: foundations for physical therapy in Rehabilitation. Gaithersburg, MD: aspen Publications, 2000:1-31.

11. Hubbard IJ, Parsons MW, Neilson C, et al. Task-specific training: evidence for and translation to clinical practice. Occup Ther Int 2009:16:175-89.

12. French B, Thomas LH, Leathley MJ, et al. Repetitive task training for improving functional ability after stroke. Cochrane Database Syst Rev 2007;17:CD006073.

13. Sakzewski L, Gordon A, Eliasson AC. The state of the evidence for intensive upper limb therapy approaches for children with unilateral cerebral palsy. J Child Neurol 2014;29:1077-90.

14. Thorpe DE, Valvano J. The effects of knowledge of performance and cognitive strategies on motor skill learning in children with cerebral palsy. Pediatr Phys Ther 2002;14:2-15.

15 Thelen E, Smith L. Theoretical Models of Human Development (chapter 6). John Wiley and Sons. London: Dynamic Systems Theories, 2007.

16. Morgan C, Darrah J, Gordon AM, et al. Effectiveness of motor interventions in infants with cerebral palsy: a systematic review. Dev Med Child Neurol 2016;58:900-9.

17. Tinderholt Myrhaug $\mathrm{H}$, Østensjø S, Larun L, et al. Intensive training of motor function and functional skills among young children with cerebral palsy: a systematic review and meta-analysis. BMC Pediatr 2014;14:294.

18. Martin L, Baker R, Harvey A. A systematic review of common physiotherapy interventions in school-aged children with cerebral palsy. Phys Occup Ther Pediatr 2010;30:294-312.

19. Darrah J, Hickman R, O'Donnell M, et al. AACPDM Methodology to develop systematic reviews of treatment interventions (Revision 1.2). 2008 https://www.aacpdm.org/UserFiles/file/systematic-reviewmethodology.pdf (accessed 21 Oct 2016).

20. Higgins JPT, Green S. Cochrane Handbook for Systematic Review of Interventions. 2011 https://www.cochrane-handbook.org (accessed 21 Oct 2016)

21. Muratori LM, Lamberg EM, Quinn L, et al. Applying principles of motor learning and control to upper extremity rehabilitation. $J$ Hand Ther 2013;26:94-103.

22. Steenbergen B, van der Kamp J, Verneau M, et al. Implicit and explicit learning: applications from basic research to sports for individuals with impaired movement dynamics. Disabil Rehabil 2010;32:1509-16.

23. Hoffmann TC, Glasziou PP, Boutron I, et al. Better reporting of interventions: template for intervention description and replication (TIDieR) checklist and guide. BMJ 2014;348:g1687.

24. Guyatt GH, Oxman AD, Vist GE, et al. GRADE: an emerging consensus on rating quality of evidence and strength of recommendations. BMJ 2008;336:924-6.

25. Bleyenheuft $\mathrm{Y}$, Arnould $\mathrm{C}$, Brandao MB, et al. Hand and arm bimanual intensive therapy including lower extremity (HABIT-ILE) in Children with Unilateral Spastic Cerebral Palsy: a Randomized Trial. Neurorehabil Neural Repair 2015;29:645-57.

26. Declerck M, Verhuel M, Daly D, et al. Benefits and enjoyment of a swimming intervention for Youth with Cerebral Palsy: an RCT Study. Ped Phys Ther 2016:28.

27. Bar-Haim S, Harries N, Nammourah I, et al. Effectiveness of motor learning coaching in children with cerebral palsy: a randomized controlled trial. Clin Rehabil 2010;24:1009-20.

28. Grecco LA, Zanon N, Sampaio LM, et al. A comparison of treadmill training and overground walking in ambulant children with cerebral palsy: randomized controlled clinical trial. Clin Rehabil 2013;27:686-96

29. Kumban W, Amatachaya S, Emasithi A, et al. Effects of task-specific training on functional ability in children with mild to moderate cerebral palsy. Dev Neurorehabil 2013;16:410-7.

30. Swe NN, Sendhilnnathan S, van Den Berg M, et al. Over ground walking and body weight supported walking improve mobility equally in cerebral palsy: a randomised controlled trial. Clin Rehabil 2015;29:1108-16.

31. Capio CM, Sit CHP, Eguia KF, et al. Fundamental movement skills training to promote physical activity in children with and without disability: A pilot study. J Sport Health Sci 2015;4:235-43.

32. Hemayattalab R, Arabameri E, Pourazar M, et al. Effects of self-controlled feedback on learning of a throwing task in children with spastic hemiplegic cerebral palsy. Res Dev Disabil 2013;34:2884-9.

33. Ketelaar M, Vermeer A, Hart $\mathrm{H}$, et al. Effects of a functional therapy program on motor abilities of children with cerebral palsy. Phys Ther 2001;81:1534-45. 
34. Hemayattalab R, Rostami LR. Effects of frequency of feedback on the learning of motor skill in individuals with cerebral palsy. Res Dev Disabil 2010;31:212-7.

35. Löwing K, Bexelius A, Brogren Carlberg E. Activity focused and goal directed therapy for children with cerebral palsy--do goals make a difference? Disabil Rehabil 2009;31:1808-16.

36. Sorsdahl AB, Moe-Nilssen R, Kaale HK, et al. Change in basic motor abilities, quality of movement and everyday activities following intensive, goal-directed, activity-focused physiotherapy in a group setting for children with cerebral palsy. BMC Pediatr 2010;10:26.
37. Russell DJ, Rosenbaum PL, Wright M, et al; Gross Motor function measure (GMFM-66 \& GMFM-88) User's Manual. London: Mac Keith Press, 2013.

38. Damiano DL, Gilgannon MD, Abel MF. Responsiveness and uniqueness of the pediatric outcomes data collection instrument compared to the gross motor function measure for measuring orthopaedic and neurosurgical outcomes in cerebral palsy. J Pediatr Orthop 2005;25:641-5.

39. Löwing K, Bexelius A, Carlberg EB. Goal-directed functional therapy: a longitudinal study on gross motor function in children with cerebral palsy. Disabil Rehabil 2010;32:908-16. 